\title{
Influence of the Presence and Type of Fragrance on the Sensory Perception of Cosmetic Formulations
}

\author{
Gisele Mara Silva Gonçalves $^{1 *}$, Silvana Mariana Srebernich ${ }^{2}$, Bartira Guiçardi Vercelino ${ }^{1}$ \\ and Bruna Melli Zampieri ${ }^{1}$ \\ ${ }^{1}$ Faculdade de Ciências Farmacêuticas; Pontifícia Universidade Católica de Campinas; Campinas - SP - Brasil. \\ ${ }^{2}$ Faculdade de Nutrição; Pontifícia Universidade Católica de Campinas; Campinas - SP - Brasil
}

\begin{abstract}
The aim of the present study was to evaluate the sensory assessments of identical cosmetic formulations with and without fragrance to investigate not only the acceptance but also how different fragrances affected their attributes, such as skin feel, tackiness and spreadability. Three gel and three cream formulations with and without two types of fragrance, fennel and sweet flowers, were assessed for various attributes. The presence and type of fragrance used affected the testers' perception of some attributes, showing that the influence of this component should not be disregarded. Apparently, a consumer's reaction to a cosmetic product is not only based on its efficacy but also on how its attributes are perceived, such as appearance, skin feel and smell.
\end{abstract}

Key words: Cosmetics, Development, Fragrance, Olfactory memory, Sensory

\section{INTRODUCTION}

Olfaction is very important to humans and has been extensively studied for better elucidation of its mechanisms and numerous applications, from the clinical diagnosis of diseases to industrial and technological applications (Lundström et al. 2011).

The majority of cosmetics, toiletries, households and laundry products contain fragrance (Bridges 2002), since they influence the purchase decision and product acceptance by the consumers. Fragrances are volatile or semi-volatile compounds; their formula are considered trade secrets and components that make up the fragrance portion of the product are not revealed on the labels.

The cosmetic composition can modify the sensory attributes and influence the consumer acceptance.
The combination of substances influences the initial feel, how the formulation spreads on the skin, whether and how fast it is absorbed and how the skin feels after the use (Lodén et al. 1992). Parente et al. (2008) characterized the sensory properties of various emollients through quantitative descriptive analysis.

Lukic et al. (2011) evaluated the emulsions containing as the only difference being the type of emollient used. It was observed that spreadability, texture and slippersiness were different for each formulation.

Selecting the correct fragrance for a new product becomes important because it can interfere directly in the purchase decision of the product. Milotic (2006) reported that the changes in the aroma of the product or packaging could affect the purchase decision considering that the fragrance was a basic factor in consumer's choice.

*Author for correspondence: gmsg@puc-campinas.edu.br 
People have very distinctive interpretations of the events they experience every day and each event is interconnected with the sensations felt during that particular event. Then, researchers ask if there was some sensory memory that interconnected experiences with the sensations felt in the environment the event occurred. Some authors have stated that such a memory did exist and termed it as autobiographical memory. There are situations in which an individual tends to correlate a certain odor with an affective phenomenon, or situations that seem odorless, or those in which odors are interpreted ambiguously or distortedly because of, for example, neurological disorders (Finkelmeyer et al. 2010, Gaygen and Hedge 2009, Lawless 1999, Lunström et al. 2011, Sela and Sobel 2010, Stevenson 2009, Sulmont-Rosse et al. 2005).

During research and development, formulators need to ensure the safety and efficacy of the products in order to meet the standards established by the cosmetic industry regulators, such as the Food and Drug Administration in the USA or the National Health Surveillance Agency in Brazil. The formulator must also consider the acceptance of the product by the consumers, since the cosmetics are over-the-counter items sold in a constantly growing market that generates billions of dollars in revenue each year (ABIHPEC 2011) and consequently results in intense competition among manufacturers.

The objective of this study was to determine if the presence or type of fragrance in the gel or cream formulations had any influence on how healthy individuals perceived them, and consequently, on how well they were accepted and testers' preference.

\section{MATERIALS AND METHODS}

This study was approved by the Ethics Committee for Research on Human Beings of the Pontifical Catholic University of Campinas, protocol number 680/00.

\section{Casuistic}

Fifty healthy nonsmokers aged 18 to 50 years of both genders agreed to participate in the study. They were all consumers of cosmetic products. The participants were asked not to eat anything at least 30 minutes before the sensory tests to avoid the interference with the results. They signed a free and informed consent form, which included the objectives of the study and information that they would be testing skin moisturizers.

\section{Studied formulations}

Two different pharmaceutical forms were selected for the sensory tests: gel and cream. The components were acquired directly from a pharmaceutical raw materials, excipients and ingredients supplier in the city of Campinas, SP, Brazil, and are listed by their INCI names (International Nomenclature of Cosmetic Ingredients).

The oil-in-water $(\mathrm{o} / \mathrm{w})$ cream was prepared with $8.0 \%$ cetearyl alcohol / sodium cetearyl sulfate (thickener and emulsifier), 5\% propylene glycol (humectant), $0.8 \%$ of a phenoxyethanol, methylparaben, ethylparaben, propylparaben, butylparaben and isobutylparaben mixture (preservatives), $2 \%$ isopropyl myristate (emollient), $0.4 \%$ propolis ethanol extract (active ingredient) and $83.5 \%$ of purified water.

The gel was prepared with $1.5 \%$ hydroxyethylcellulose (water-soluble polymer), 5\% propylene glycol (humectant), $0.8 \%$ of a phenoxyethanol, methylparaben, ethylparaben, propylparaben, butylparaben and isobutylparaben mixture (preservatives), $0.4 \%$ propolis ethanol extract (active ingredient) and $0.92 \%$ of purified water.

The lots of $300 \mathrm{~g}$ of each formulation were prepared and divided into three portions of $100 \mathrm{~g}$ each. A $0.25 \%$ of a fine, sweet, floral fragrance was added to the first portion (fragrance A); the same amount of fennel fragrance were added to the second portion (fragrance B) and the third portion remained fragrance-free. Hence, a total of six formulations were made, three cream formulations and three gel formulations, each in three varieties: sweet floral fragrance, fennel fragrance and fragrance-free.

\section{Sensory analysis}

Twenty-five untrained testers were assigned to assess the gel formulations and twenty-five to assess the cream formulations-, performing the affective testing. The study protocol was designed by the researchers.

The test was done in white, ventilated sensory analysis booths free from strong odors and with red lighting to prevent the testers from identifying 
or differentiating the products based on the color. The booths allowed the testers to sit comfortably. They were allowed to remain inside for as long as they wanted.

Before the tests began, the samples were placed inside the plastic cosmetic jars and randomly numbered with three-digit codes. All the samples were at room temperature, that is, approximately $25^{\circ} \mathrm{C}$. The testers made themselves comfortable inside the booths and were given the samples in random order with the assessment forms. The form instructed the tester to apply a small amount of one of the products on the back of his hand and analyze its characteristics. The same procedure was repeated for the second and third formulations. The testers were instructed to apply each product on a different area of the back of the hand so one product would not be applied on top of another product. At the end of the test, the volunteer used a light to signal to the researchers that he was done and was allowed to leave the booth without interacting with other testers.

The questionnaire contained the following questions: if the product would or not be effective; if the samples were pleasant or not; if the samples were similar to common, neutral or special products; if the formulations were greasy, whitened the skin on application, were easy to spread, were easily absorbed; what kind of odor the formulation had; which formulation he would use; and, to finish, the questionnaire asked the tester to compare the formulations.

\section{Analysis and interpretation}

The data in the questionnaires were tabulated and discussed.

\section{RESULTS AND DISCUSSION}

Figure 1 shows the number of answers regarding the perceived efficacy of the formulations. In general, the testers believed that all the formulations were effective, since out of 25 testers, 13 stated that all the formulations seemed effective and only a minority stated that the formulations would not be effective. Perceived efficacy is subjective. However, one-third of the testers deemed the fragrance-free gel to be ineffective; no other formulation had this many testers doubting its efficacy. Therefore, the absence of fragrance might have been influenced the testers and the perception of the initial efficacy might be related to the fragrance. According to Milotic (2006), the role of fragrance in consumer satisfaction is often underestimated.
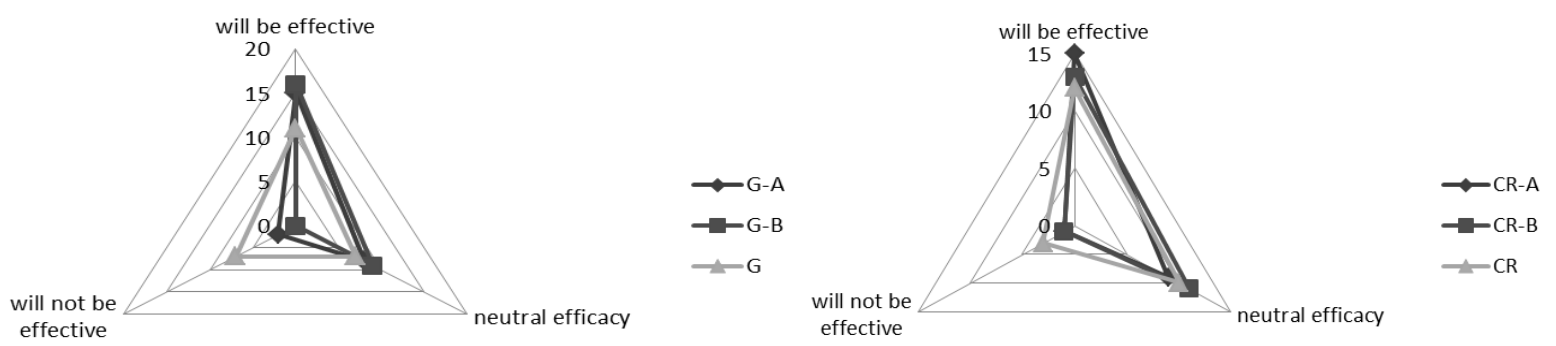

Figure 1 - Perceived efficacy of the formulations. (CR) cream formulation; (G) gel formulation; (A) sweet floral fragrance; (B) fennel fragrance $(n=25)$.

Figure 2 presents the answers for the pleasantness of the formulations. It showed that most testers classified the formulations as pleasant. According to Milotic (2006), when a product contains a pleasant fragrance, it generates a positive attitude as the desire of continuing handling and smelling the product and breathing tends to be enhanced in the presence of a pleasant odor.

The answers regarding the cream and gel formulations with fragrance were similar and apparently fragrance had little influence on this attribute. 

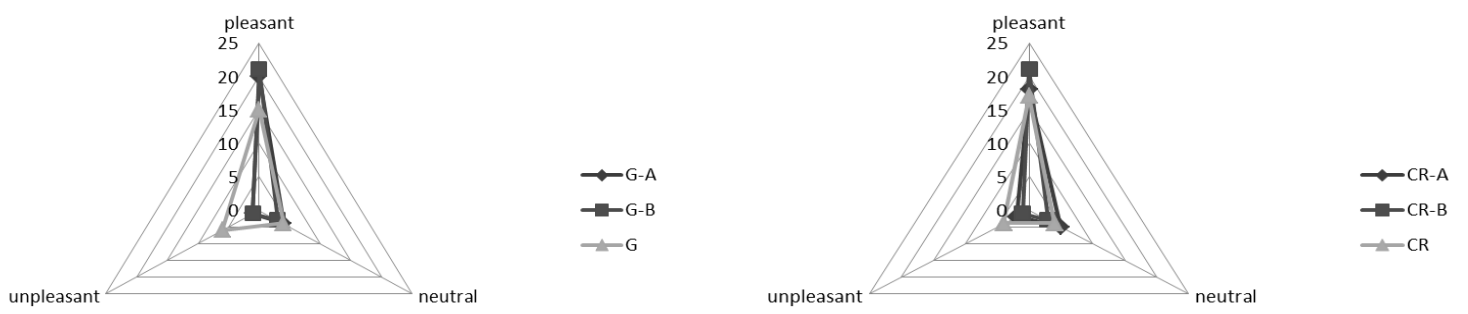

Figure 2 - Perceived pleasantness of the formulations. (CR) cream formulations; (G) gel formulations; (A) sweet floral fragrance; (B) fennel fragrance $(n=25)$.

When the testers were asked to classify the formulations as special, neutral or common (Fig. 3 ), the fragrance-free cream formulation was considered the most common, since 19 testers considered it neutral or common and only six considered it special. On the other hand, the cream formulation with fennel fragrance was considered special by 12 testers. All other four formulations were considered common by some and special by others, therefore, not standing out one way or the other. In fact, one feature that distinguishes products perceived as special is the use of exclusive fragrances. However, fennel essence is widely used in Brazilian cosmetics, therefore not exclusive. Even so, the cream formulation containing it was still classified by most testers as the most special, possibly because it was milder and more delicate than the sweet floral fragrance. Additionally, the testers were probably also influenced by their personal taste or autobiographical memory when they classified it. This result led to a reflection about the advertising of "exclusive" or "special" products, aimed at higher-income individuals. Advertising is essential for constructing an idea of specialness and aggregating value to a product. When the connection between the advertising and product or the first impression a product makes is broken, it is possible that the consumer no longer recognizes the specialness of the product. In a way, this disconnection was made in the present study and the most popular essence was also the testers' favorite. Therefore, it is clear that a fragrance can affect a person's opinion of a cosmetic product. The results for the gel formulation were similar; hence, pharmaceutical form had no impact on the distinctiveness of a product.

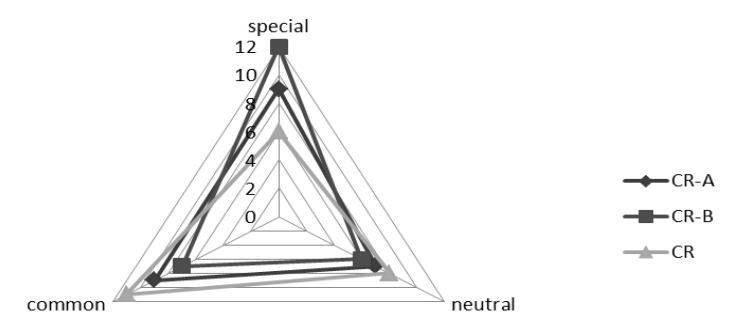

Figure 3 - Perceived distinctiveness of the formulations. (CR) cream formulations; (G) gel formulations; (A) sweet floral fragrance; (B) fennel fragrance $(n=25)$.

Figure 4 shows the perceived level of greasiness of the formulations. Most testers considered the cream formulation with the sweet floral fragrance either greasy or very greasy (14 answers) and the gel formulation with the fennel fragrance not greasy or mildly greasy (17 answers). Considering that the amount of fragrance used in all but the fragrance-free formulations was identical, it was evident that the different degree of greasiness perceived by the testers was based on their personal preference for one or another fragrance, which explained the diverse answers. It was 
possible that the olfactory memory and personal preference of the testers were determinants of perceived greasiness. It would be critical to study how a product performs in a product testing panel before launching it because, regardless of how effective or safe a product is, if the consumer does not like its fragrance, he may have a completely different perception of the product, making the product fail miserably.

Considering the fragrances used in this study, fennel could be considered smoother and more refreshing than the floral, more striking and sweety, which might have affected the perception of the testers on the greasiness. Therefore, the fragrances are the subject of a complex discussion because its interpretation varies with context, and external factors can influence since the

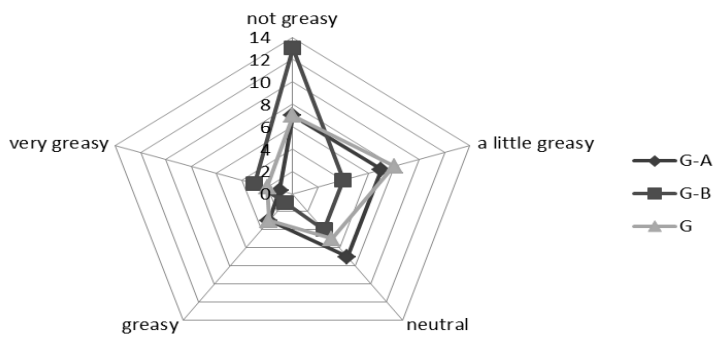

recognition of a particular fragrance as its personal interpretation, linked also to the sensory memory (Milotic 2006).

Also, when it refers to a sales appeal, there must be more elements which promote the desired comprehension of the fragrance used in the product such as colors and images, for example. In this study, no evidence was offered to the testers, which favored the immediate recognition of the fragrances used since it was carried out in sensory analysis cabins using red light and the products were presented in neutral packaging. Thus, the results were due to an overall assessment of the product, where one element (odor) might have influenced another one (greasiness, for example), what was desired and consisted in the aim of this work.

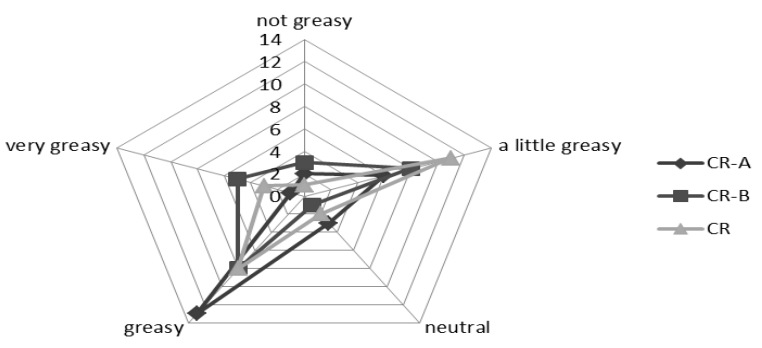

Figure 4 - Perceived greasiness of the formulations. $(C R)$ cream formulations; $(G)$ gel formulations; (A) sweet floral fragrance; $(B)$ fennel fragrance $(n=25)$.

In relation to the whitening of the skin, that is, foamed on application, there were no differences between the formulations with and without fragrance. Some testers (9 to 10) thought the cream formulations caused whitening and most (15 to 16) thought it did not. Foam on application is caused by the presence of an emulsifier and absence of foam inhibitors. Gel formulations did not contain emulsifiers and indeed, all the testers stated that skin whitening did not occur. Expectedly, the fragrances had no influence on this parameter because this parameter was a visual attribute, which would hardly be influenced by the aroma of the product.

Spreadability is a very important attribute because it affects the performance of a product during the application. Figure 5 showed that the spreadability of all formulations was similar; all were considered easy to spread. The formulation considered easiest to spread was the gel formulation with the fennel fragrance, which was considered easy to spread by the highest number of the testers (18). Spreadability can be tested mechanically during the product development using two flat, circular glass plates with standard diameter and thickness. A known volume of the product is put on one plate and the other plate is allowed to rest on top of it. Spreadability is given as the final diameter of the sample sandwiched between the two plates after one minute (Borghetti and Knorst 2006). Gel and cream formulations, in general, present pseudoplastic behavior. This means that apparent viscosity decreases as tension increases. This behavior is desirable in cosmetic products because it facilitates application of the product on the skin (Gonçalves and Maia Campos 2009). In this case, fragrance seemed to have affected the results since the spreadability of the formulations should be almost identical, especially those with the fragrance. The oily nature of 
fragrances could facilitate product spreading on the skin.

Mehling et al. (2010) compared the formulations containing or not, different emollients $(2 \%)$ and considered that the major role of emollients in leave-on formulations was the creation of a pleasant and elegant skin feel. The authors observed that emollient containing formulations with their emollient--free surfactant base, showed positive effect of the emollients on the skin feel. Consistent with this performance, a higher amount of adsorbed emollient correlated well with a more pleasant skin-feel. The sensory assessments confirmed the positive effect of added emollients on the skin-feel after the rinse-off application. As expected, a higher amount of adsorbed emollient correlated with an even more positively perceived skin feel. Gorcea and Laura (2010) compared four ester emollients applied directly on the skin and they concluded that this approach could provide informative guidance for the cosmetic formulator in selecting various specific emollient esters for personal care and topical pharmaceutical formulations, and further predicting a product's distinct sensory characteristics.

In this study, fragrance seemed to have affected the results since the spreadability of the formulations should be almost identical, especially those with fragrance, because the amount used was small and below the usual concentration of emollients. This would be with the fact that the oily nature of fragrances could facilitate product spreading on the skin in a manner similar to the softeners, which were also substances with oily characteristics.

In relation to the concentration used, as for the fragrance, the concentration should be fairly small, since each scent has an optimal level of concentration (the most preferred concentration). After a point, as the intensity of a scent increases, its pleasantness decreases. An odour in low concentrations may be perceived as quite pleasant while the same odour in high concentrations can be unpleasant (Milotic 2006).

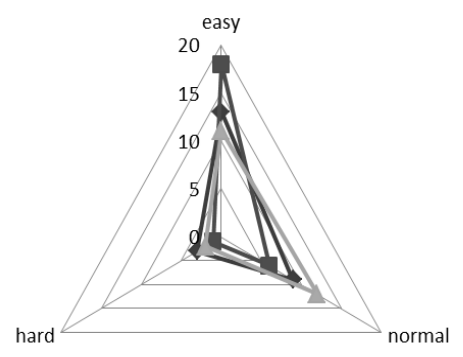

Figure 5 - Perceived spreadability of the formulations on the skin. (CR) cream formulations; $(G)$ gel formulations; (A) sweet floral fragrance; (B) fennel fragrance $(n=25)$.
In relation to the perceived penetration of the formulations according to the testers, the fragrance-free formulations presented slow to average penetration, while those with fragrances were considered slightly better. The fragrances clearly influenced this attribute and differentiated the formulations with from those without fragrance but it was not possible to determine which fragrance promoted better__perceived penetration.

Figure 6 shows how the testers perceived the aroma of the fragrances. The cream formulation with the fennel fragrance received the highest number (22) of positive comments. The cream formulation with sweet floral fragrance was also well accepted, receiving 18 positive comments, that is, its smell was considered either good or pleasant. The fragrance-free formulation received the highest diversity of comments, including the highest number of testers disliking its smell. Meanwhile, the fragrance-free gel formulation was not popular among the testers since most testers (18) considered its smell from regular to terrible. On the other hand, the smell of the gel formulations with fragrance was considered from pleasant to good by most testers (16 liked the sweet floral fragrance and 18 liked the fennel fragrance). Since aroma was an attribute that 
depends on personal preference, this diversity of answers and preference for formulations with fragrance were expected. Personal preference influenced the results, making aroma very

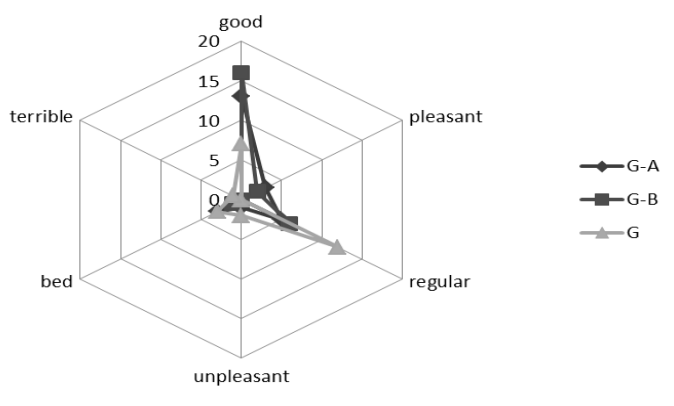

important. Its influence indicates that fragrance must be selected carefully as it may aggregate quality to a product and determine consumer preference.

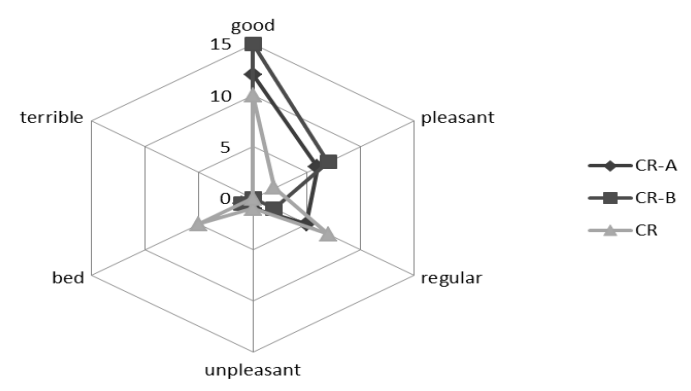

Figure 6 - Formulation odor classification. (CR) cream formulations; (G) gel formulations; (A) sweet floral flagrance; (B) fennel flagrance $(\mathrm{n}=25)$.

When the testers were asked if they would use any of the formulations they generally preferred the one with fennel fragrance (18 answers to cream and 20 answers to gel). This was certainly related to the aroma of the formulation and reflected the personal preference of each tester. Additionally, most of the testers would not use the fragrancefree formulations. Once again, the presence and type of fragrance influenced the testers' preferences, reflecting its importance.

Figure 7 shows the preference of the testers for the different formulations. The fennel fragrance was the favorite among the cream formulations and the gel formulations with fragrance prevailed over the fragrance-free gel formulation. When the testers were asked which formulation penetrated best, the replies did not vary too much. In this case, in the two extremes were the fragrance-free gel, indicated by four testers, and the gel formulation with fennel fragrance, indicated by ten testers. As before, it was not possible to determine the best fragrance.

The fragrance-free gel formulation was considered the greasiest gel by more testers (13) than any other formulation. This was interesting because this formulation did not have any greasy component in its composition, not even an essence. It seemed that an attribute interfered with the other ones, that is, the absence of a fragrance had a negative impact on how the testers perceived the other attributes, which were unrelated to smell. The fragrance-free cream formulation was also considered the greasiest among the cream formulations according to ten testers.

These results corroborated those of Dermatte et al. (2006) who correlated the sense of touch with the sense of smell and demonstrated the existence of a cross-modal interaction between olfaction and touch.
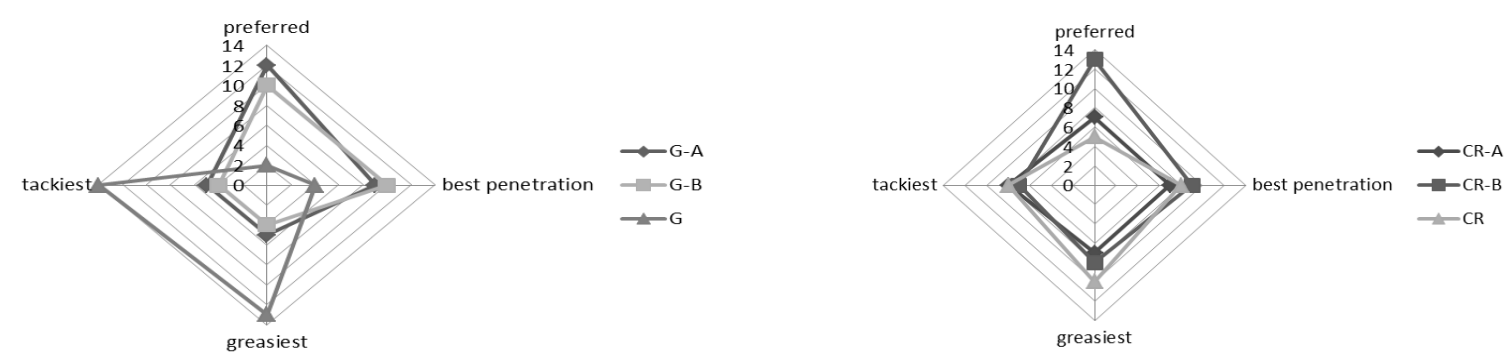

Figure 7 - Preferred formulations and formulations that presented the best perceived penetration, greasiness and tackiness. (CR) cream formulations; (G) gel formulations; (A) sweet floral flagrance; (B) fennel flagrance $(n=25)$. 
These authors also mentioned that there were effects exerted by stimuli presented in one sensory modality on people's responses to stimuli presented in another modality could be due to a process of associative learning.

In the present study, the fragrance-free gel formulation was also considered the tackiest by most testers (14). The other formulations had similar perceived tackiness since the number of testers that indicated each as tackiest was similar. This attribute could have been influenced by two factors: personal preference and absence of fragrance. Since fragrances have an oily nature, they could indeed reduce the tackiness slightly. Therefore, despite the results, it was not possible to determine if fragrance interfered because of its aroma, because of its oily nature or both. An alternative to this problem could be to add to the non-fragrance product the same amount of a mineral-oil, with no essence, for example.

The objective of this study was to compare the sensory properties of gel and cream formulations with different aromas to determine how fragrance affected the global perception of the product and specifically, the skin feel of the product when it was applied on the back of the hand. Studies with such objectives are scarce in the scientific literature.

Two pharmaceutical forms were tested, a hydrophilic gel and an oil-in-water cream. The gels consist basically of a hydrophilic polymer dispersed in water, plus preservatives and humectant, that is, a product consisting of hydrophilic substances only, while creams contain hydrophilic and hydrophobic substances, such as a superior fatty alcohol (cetearyl alcohol) and an emollient ester (isopropyl myristate). Hence, perceived spreadability, tackiness, greasiness and skin whitening of the two forms could differ, regardless of smell.

The only thing that distinguished the formulations was their aroma. A fragrance, currently being widely used in Brazil in the cosmetics and toiletries, called fennel, was selected for the present study, along with a lesser known sweet floral fragrance whose aroma was more pronounced and prominent. The intention was to test two aromas that were very different from each other to verify if either one would have any impact on the testers' perception of the other attributes of the formulations.
The questionnaire was developed for untrained testers, that is, people that had strong preferences. The words used in the questionnaire were very simple and ordinary so that it could be easily and unambiguously understood. All the answers were based on the application of each product by the tester in different areas of the back of his hand, being careful not to apply one product over another. First, the testers assessed each formulation individually and then compared them.

The results showed that the presence and type of fragrance influenced how the formulations were perceived since the testers reported differences among their attributes despite their nearly identical composition. According to Drake (2007), sensory analysis is a tool to measure the quality and success of a product.

Since the testers in the present study did not undergo any previous training, only an initial explanation, one may say that they represent the final consumer. When they were asked about their preference, that is, which formulation they liked more or which they would use, their answers were possibly influenced by their personal preferences, which was automatically associated with the olfactory memory of each individual. Hence, olfactory responses were mediated by the familiarity of an odor and, consequently, sensory perception would relate to the olfactory response.

The reaction to the fennel fragrance in the present study was positive since the testers preferred the formulations with it. The very delicate smell of this fragrance could explain its popularity. However, if at that time, the testers were looking for something with a delicate smell, the fragrancefree formulations could have been well--accepted too. However, they were the least popular. Since this fragrance is extensively used in all kinds of cosmetic products, the hypothesis of olfactory memory is plausible. Since the sweet floral fragrance is not only less known but also very pronounced, this may explain its lower acceptability, as it is a very sweet fragrance. Furthermore, some of the testers were males, and they rarely used the products with sweet smells since sweetness has been associated with forfemale products.

Another factor to be considered was that some attributes were more influenced by the fragrance (greasiness and spreadability) than others (skin whitening). Thus, when products are being 
developed, the formulator needs to make adjustments in the formulation to improve its attributes, and these adjustments include choosing another fragrance, emollients and thickeners, among others, in order to improve the texture of the product on the skin, minimize whitening and improve the sensation caused by the product on application. Many silicone fluids are among the numerous substances that are being widely used for improvement of the sensory properties of cosmetics (Baquerizo et al. 1999, Brummer and Godersky 1999, Roso and Brinet 2005).

Gonçalves et al. (2011) studied the stability and sensory attributes of formulations containing the extract of propolis with or without tocopheryl acetate and found that in the sensory analysis performed with 28 judges, the formulation containing propolis extract in combination with tocopheryl acetate was preferred, as compared with the cream and cream base containing only propolis extract or tocopheryl acetate. The study was carried out due to the fact that propolis presented unique odor, which could interfere with the acceptance of the product, which did not occur. Moskowitz (1986) studied the sensory segmentation of fragrance preference and found plenty of evidence that there was personal preference for certain fragrances.

The results of the present study proved that the sensory perception of a topical product could be influenced by its fragrance. The formulator must focus not only on the efficacy and safety of formulations but also on the sensory testing, and select the best fragrance for each product. According to Porcherot et al. (2010), each culture may require a specific questionnaire for assessing the sensations produced by different fragrances, since the best terminology to define the sensations may vary from place to place or from country to country.

Finally, it is known that aromatherapy has been used as a means for improving the well-being; therefore, it is used as a complementary therapy for anxiety, pain, to improve quality of sleep, among other specific objectives, many times together with specific massages. Although its action is not always proven, there is evidence that it helps to obtain the expected results (Bagetta et al. 2010, Barclay et al. 2006, Kritsidima et al. 2010). Hence, essential oils may improve people's mood and even health. Studies on food have made great contributions to the knowledge about human perception of products. Labbe et al. (2012) studied how consumers perceived packaged foods based on the packaging and found that the naturalness of the packaged food can be reasonably well determined by its packaging.

The use of this type of knowledge may be extended to the cosmetic and perfume industry. Moreover, sensory analysis may be used as a tool for improving the quality of pharmaceutical drugs (Fregonezi-Nery et al. 2002).

\section{CONCLUSIONS}

In general, the perception of the testers regarding the sensory characteristics of the cream and gel formulations was influenced by the presence or absence of fragrance. The formulations with fragrance were preferred by most individuals to the ones without. The type of fragrance used in both the gel and cream formulations affected the testers' sensory perception of them. Furthermore, the testers preferred the fennel fragrance to the sweet floral fragrance. Skin feel is an important sensory area for cosmetic products. Therefore, consumers' reactions to a cosmetic product are not only based on the product efficacy but also on subjective factors, such as skin feel and aroma.

\section{ACKNOWLEDGMENTS}

The authors thank the volunteers who agreed to participate in this study and the Pontifical Catholic University of Campinas for the support.

\section{REFERENCES}

ABIHPEC Panorama do Setor [homepage on the internet]. São Paulo: ABIHPEC. [cited 2011 Jun. 10].Available in http://www.abihpec.org.br/wpcontent/uploads/2012/04/Panorama-do-setor-20112012-03-DEZ-2012.pdf.

Bagetta G, Morrone LA, Rombolà L, Amantea D, Russo R, Berliocchi L, et al. Neuropharmacology of the essential oil of bergamot. Fitoterap. 2010; 81(6): 453-461.

Baquerizo I, Gallardo V, Parera A, Ruiz MA. Development, formulation, and effectiveness testing of a silicone-based barrier-type hand cream. Drug. Dev. Ind. Pharm. 1999; 25(12):1259-1265. 
Barclay J, Vestey J, Lambert A, Balmer C. Reducing the symptoms of lymphoedema: Is there a role for aromatherapy? Eur. J. Oncol. Nurs. 2006; 10(2): 140-149.

Bridges B. Fragrance: emerging health and environmental concerns. Flavour Fragr. J. 2002; 17(5): 361-371.

Borghetti GS, Knorst MT. Desenvolvimento e avaliação da estabilidade física de loções O/A contendo filtros solares. Rev. Bras. Cienc. Farm. 2006; 42(4): 531-537.

Brummer R, Godersky S. Rheological studies to objectify sensations occurring when cosmetic emulsions are applied to the skin. Coll. Surf. A: Physicochem. Eng. Asp. 1999;152(1-2): 89-94.

Dermatte ML, Sanabria D, Sugarman R, Spence CH. Cross-Modal interactions between olfaction and touch. Chem. Sens. 2006; 31(4): 291-300.

Finkelmeyer A, Kellermann T, Bude D, Nießen T, Schwenzer M, Mathiak K, Reske M. Effects of aversive odour presentation on inhibitory control in the Stroop colour-word interference task. BMC Neurosci. 2010; 11:131-138.

Fregonezi-Nery MM, Prudencio-Ferreira SH, Kedor EMR, Bacarat MM, Brinholi FF. Sensory evaluation of albendazole suspensions. Braz. Arch. Biol. Technol. [online] 2002; 45(4): 457-463.

Gaygen DE, Hedge A. Effect of Acute Exposure to a Complex Fragrance on Lexical Decision Performance. Chem. Sens. 2009; 34(1): 85-91.

Gonçalves GMS, Maia Campos PMBG. Shelf life and rheology of emulsions containing vitamin $\mathrm{C}$ and its derivatives. Rev. Ciênc. Farm. Básica. Apl., 2009; 30(2): 217-224.

Gonçalves GMS, Srebernich SM, Souza JAM. Stability and sensory assessment of emulsions containing propolis extract and/or tocopheryl acetate. Braz. J. Pharmac. Sci. 2001; 47(3): 585-592.

Gorcea M, Laura D. Evaluating the physiochemical properties of emollient esters for cosmetic use. Cosmet. Toil. 2010; 12: 26-33.

Kritsidima M, Newton T, Asimakopoulou K. The effects of lavender scent on dental patient anxiety levels: a cluster randomized-controlled trial. Community Dent. Oral Epidemiol. 2010; 38(1): 8387.

Labbe D, Pineau N, Martin N. Measuring consumer response to complex perception: scaling vs. categorization task. Food. Qual. Pref. 2012; 23(2): 134-137.
Lawless RT. Descriptive analysis of complex odors: reality, model or illusion? Food. Qual. Pref. 1999; 10(4-5): 325-332.

Lukic M, Jaksic I, Krstonosic V, Cekic N, Savic S. A combined approach in characterization of an effective w/o hand cream: the influence of emollient on textural, sensorial and in vivo skin performance. Int. J. Cosmet. Sci. 2012; 34(2): 140-149.

Lunström JN, Boesveldt S, Albrecht J. Central Processing of the Chemical Senses: An Overview. ACS Chem. Neurosci. 2011; 2(1): 5-16.

Mehling A, Haake HM, Poly W. Differential deposition of emollients from tripartite formulation systems. Int. J. Cosmet. Sci. 2010; 32(2): 117-125.

Milotc D. The impact of fragrance on consumer choice. J. Consumer Behaviour. 2006; 3(2): 179-191.

Moskowitz HR. Sensory segmentation of fragrance preferences. J. Soc. Cosmet. Chem. 1986; 37(4): 233 247.

Parente ME, Gámbaro A, Solana G. Study of sensory properties of emollients used in cosmetics and their correlation with physicochemical properties. I. J. Cosmet. Sci. 2005; 27(6): 354.

Porcherot C, Delplanque S, Raviot-Derrien S, Calvé BL, Chrea C, Gaudreau N, Cayeux I. How do you feel when you smell this? Optimization of a verbal Measurement of odor-elicited emotions. Food. Qual. Pref. 2010; 21(8): 938-947.

Roso A, Brinet R. Rheology and Texture Analysis Used Together to Improve Raw Material Choices. Cosm. Toil. 2004; 119(6): 53-60.

Sela L, Sobel N. Human olfaction: a constant state of change-blindness. Exp Brain Res. 2010; 205(1):1329.

Stevenson RJ. Review: Phenomenal and access consciousness in olfaction. Conscious. Cogn. 2009; 18(4): 1004-1017.

Sulmont-Rosse C, Issanchou S, Köster EP. Odor Naming Methodology: Correct Identification with Multiple-choice versus Repeatable Identification in a Free Task. Chem. Sens.2005; 30(1): 23-27.

Received: July 20, 2011; Revised: October 11, 2011 Accepted: December 17, 2012. 